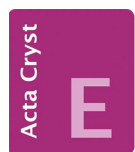

CRYSTALLOGRAPHIC COMMUNICATIONS

ISSN 2056-9890

Received 30 June 2017

Accepted 6 July 2017

Edited by O. Blacque, University of Zürich, Switzerland

Keywords: crystal structure; 5-ethynylpyrimidine derivative; $\mathrm{O}-\mathrm{H} \cdots \mathrm{N}$ hydrogen bonding; $\mathrm{C}-\mathrm{H}$... O contact.

CCDC reference: 1560599

Supporting information: this article has supporting information at journals.iucr.org/e

\section{Crystal structure of 2-chloro-5-(3-hydroxy-3- methylbut-1-yn-1-yl)pyrimidine}

\author{
Jörg Hübscher, Wilhelm Seichter and Edwin Weber*
}

Institut für Organische Chemie, TU Bergakademie Freiberg, Leipziger Strasse 29, D-09596 Freiberg/Sachsen, Germany. *Correspondence e-mail: edwin.weber@chemie.tu-freiberg.de

In the title compound, $\mathrm{C}_{9} \mathrm{H}_{9} \mathrm{ClN}_{2} \mathrm{O}$, the ethynylpyrimidine moiety displays an almost planar geometry. In the crystal, molecules are linked by $\mathrm{O}-\mathrm{H} \cdots \mathrm{N}$ and $\mathrm{C}-\mathrm{H}_{\text {pyrimidine }} \cdot \mathrm{O}$ hydrogen bonds, forming a three-dimensional supramolecular architecture.

\section{Chemical context}

The title compound, featuring a blocked acetylenic group and a chloro-substituted pyrimidine ring, is an interesting synthetic intermediate for the preparation of application-oriented solid materials including both porous coordination polymers (MacGillivray, 2010) and metal-organic frameworks (Noro \& Kitagawa, 2010). Deprotection of the acetylenic functional group and transformation of the chloro substituent, e.g. into thiol or amino groups, should result in molecular building blocks for the formation of corresponding aggregate structures (Hübscher et al., 2015; Günthel et al., 2015; Hübscher et al., 2017). Aside from this experimental preparative relevance, substituted 3-hydroxyalkynes are also of considerable interest due to their structural capacity in supramolecular interactions, giving rise to particular modes of aggregation and behavior in the solid state (Toda et al., 1983, 1985; Bourne et al., 1994). In combination with heterocyclic nitrogen donors and chlorine substitution, as in the present title compound, a structural study involving competition aspects with regard to hydrogen bonding (Wang \& Zheng, 2015) and potential halogen (Mukherjee et al., 2014) or $\pi$-electron assisted (Tiekink \& Zukerman-Schpector, 2012) interactions should be a promising field of inquiry for crystal engineering (Desiraju et al., 2012) being subject to the contacts emanating from a variety of functional groups. Thus, in this respect, the title compound could serve as a worthwhile test substance.

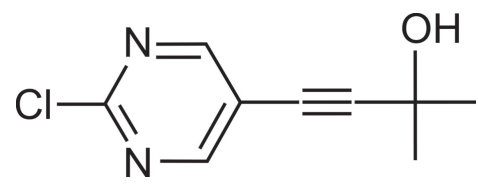

\section{Structural commentary}

A perspective view of the molecular structure of the title compound is depicted in Fig. 1. The ethynylpyrimidine moiety of the molecule is almost planar with the largest atomic distances from the mean plane being 0.015 (1) $\AA$ for atom C1 
Table 1

Hydrogen-bond geometry $\left(\AA,^{\circ}\right)$.

\begin{tabular}{lllll}
\hline$D-\mathrm{H} \cdots A$ & $D-\mathrm{H}$ & $\mathrm{H} \cdots A$ & $D \cdots A$ & $D-\mathrm{H} \cdots A$ \\
\hline $\mathrm{C} 8-\mathrm{H} 8 A \cdots \mathrm{Cl} 1^{\mathrm{i}}$ & 0.98 & 2.99 & $3.7911(14)$ & 140 \\
$\mathrm{C} 4-\mathrm{H} 4 \cdots \mathrm{O} 1^{\mathrm{ii}}$ & 0.95 & 2.45 & $3.1963(15)$ & 136 \\
$\mathrm{C} 2-\mathrm{H} 2 \cdots 1^{\mathrm{iii}}$ & 0.95 & 2.60 & $3.2816(14)$ & 129 \\
$\mathrm{O} 1-\mathrm{H} 1 \cdots \mathrm{N} 2^{\text {iv }}$ & 0.84 & 2.05 & $2.8881(13)$ & 172 \\
\hline
\end{tabular}

Symmetry codes: (i) $\quad-x+\frac{1}{2}, y-\frac{1}{2},-z+\frac{1}{2}$; $\quad$ (ii) $\quad-x+1,-y,-z+1$; $\quad$ (iii) $-x+\frac{1}{2}, y+\frac{1}{2},-z+\frac{3}{2}$; (iv) $x+\frac{1}{2},-y+\frac{1}{2}, z+\frac{1}{2}$.

and 0.013 (1) $\AA$ for atom $\mathrm{C} 4$. The $\mathrm{OH}$ group adopts a staggered arrangement with respect to the ethynyl unit and the methyl group $\mathrm{C} 9$, the $\mathrm{C} 6-\mathrm{C} 7-\mathrm{O} 1-\mathrm{H} 1$ torsion angle being $57.0^{\circ}$.

\section{Supramolecular features}

An $\mathrm{O}-\mathrm{H} \cdots \pi_{\mathrm{C} \equiv \mathrm{C}}$ hydrogen-bond type intermolecular interaction mode typical of 3-hydroxyalkyne structure units (Desiraju \& Steiner, 1999) is not present here, apparently in favor of a stronger $\mathrm{O}-\mathrm{H} \cdots \mathrm{N}$ hydrogen bond involving the hydroxy group and a pyrimidine nitrogen atom (N2). Aside from this, $\mathrm{C}-\mathrm{H}_{\text {pyrimidine }} \cdot \mathrm{O}$ hydrogen bonds are found to yield a three-dimensional supramolecular architecture (Table 1, Fig. 2). No other types of directed intermolecular contacts, including those involving the $\mathrm{Cl}$ atom or $\pi$-arene stacking, are observed. Hence, this shows that in the presence of a strong donor center such as a nitrogen atom, competing with the acetylenic moiety, the common $\mathrm{O}-\mathrm{H} \cdots \pi_{\mathrm{C} \equiv \mathrm{C}}$ hydrogen bonding is suppressed, which could be a useful finding in relation to aspects of crystal engineering.

\section{Database survey}

The title compound represents the first example of a 5-(3hydroxy-3-methylbut-1-yn-1-yl)pyrimidine. A search in the Cambridge Structural Database (CSD, Version 5.38, update February 2017; Groom et al., 2016) for compounds containing the 4-ethynylpyrimidine fragment excluding metal complexes

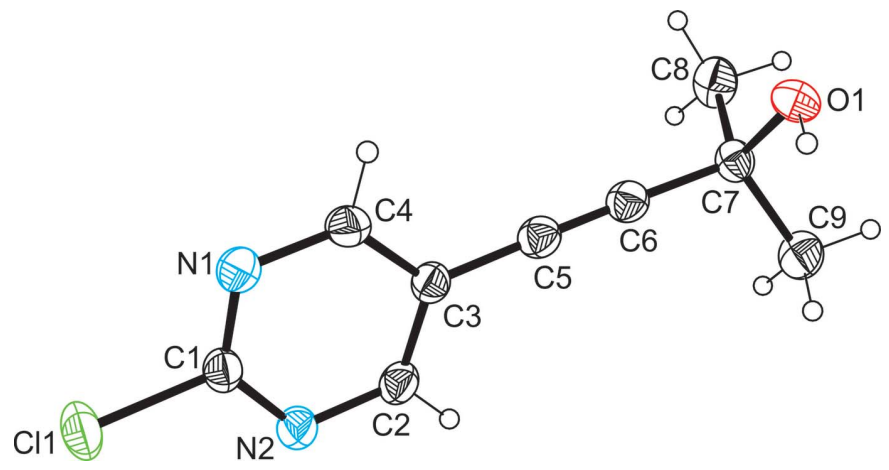

Figure 1

Perspective view of the molecular structure of the title compound including the atom-numbering scheme. Displacement parameters are drawn at the $50 \%$ probability level.

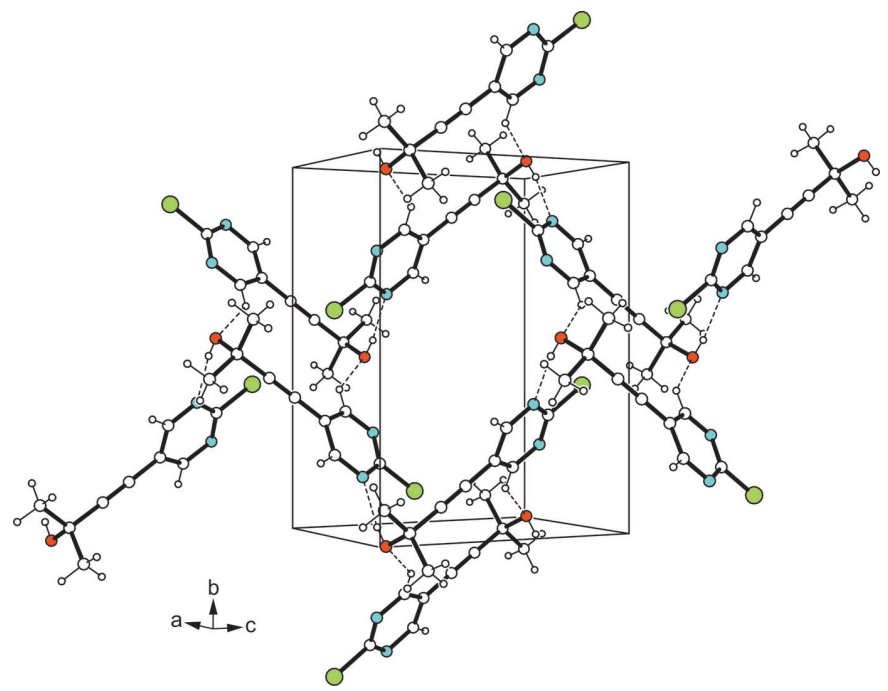

Figure 2

Packing excerpt of the title compound. Hydrogen bonds are shown as dashed lines.

and co-crystals revealed nine hits. Of particular interest is the crystal structure of 5,5'-ethyne-1,2-diylbis(2-chloropyrimidine) (refcode: PUMHIQ; Hübscher et al., 2015). In this case, the absence of a strongly coordinating donor/acceptor substituent results in poor molecular association, which is restricted to $\pi_{\text {pyrimidine }} \cdots \pi_{\text {ethyne }}$ stacking interactions.

\section{Synthesis and crystallization}

The title compound was prepared from 2-hydroxy-5-iodopyrimidine (Pérez-Palado et al., 2007) and 2-methyl-3-butyn-2ol (MEBYNOL) via a Shonogashira-Hagihara cross-coupling reaction (Sonogashira et al., 1975) as follows. 2-Chloro-5iodopyrimidine $(2.0 \mathrm{~g}, 8.4 \mathrm{mmol})$ and MEBYNOL $(0.7 \mathrm{~g}$, $8.7 \mathrm{mmol}$ ) were dissolved in a degassed mixture of dry diisopropylamine and THF (60 ml each). To this solution, the catalyst being composed of triphenylphosphine ( $2 \mathrm{~mol} \%)$, copper(I) and iodide (3 mol- $\%$ ) and trans-dichlorobis(triphenylphosphine)palladium(II) ( $2 \mathrm{~mol} \%$ ) was added. The mixture was stirred at room temperature away from light for $12 \mathrm{~h}$, then filtered over Celite and evaporated. Crystallization from $n$-hexane gave colourless crystals of the title compound on slow evaporation of the solvent (yield $1.1 \mathrm{~g}, 70 \%$; m.p. $455 \mathrm{~K}) .{ }^{1} \mathrm{H}$ NMR $\left(\mathrm{CDCl}_{3}\right): \delta_{\mathrm{H}} 8.64(2 \mathrm{H}, s, \mathrm{pyr}-\mathrm{H}), 2.67(1 \mathrm{H}, s$, $\mathrm{OH}), 1.64(6 \mathrm{H}, s, \mathrm{Me}) .{ }^{13} \mathrm{C} \mathrm{NMR}\left(\mathrm{CDCl}_{3}\right): \delta_{\mathrm{C}} 161.1$ (pyrC-4), 159.4 (pyrC-2), 117.8 (pyrC-5), 102.4 (pyr- $C \equiv \mathrm{C}$ ), 74.1 (pyr$\mathrm{C} \equiv C), 65.5\left(\mathrm{C}_{\text {quat. }}\right), 31.1\left(\mathrm{CH}_{3}\right)$. IR $(\mathrm{KBr}) v_{\text {max. }} 2240(\mathrm{C} \equiv \mathrm{C})$. GC-MS: calculated for $\mathrm{C}_{9} \mathrm{H}_{9} \mathrm{~N}_{2} \mathrm{OCl}$ (196.04), found $196[M]^{+}$. Analysis calculated for $\mathrm{C}_{9} \mathrm{H}_{9} \mathrm{~N}_{2} \mathrm{OCl}$ : C, 54.97; H, 4.61; N, 14.25; found: $\mathrm{C}, 54.81 ; \mathrm{H}, 4.56 ; \mathrm{N}, 14.05 \%$. Colourless crystals suitable for X-ray diffraction were obtained by slow evaporation of solvent from a chloroform solution. 
Table 2

Experimental details.

\begin{tabular}{|c|c|}
\hline \multicolumn{2}{|l|}{ Crystal data } \\
\hline Chemical formula & $\mathrm{C}_{9} \mathrm{H}_{9} \mathrm{ClN}_{2} \mathrm{O}$ \\
\hline$M_{\mathrm{r}}$ & 196.63 \\
\hline Crystal system, space group & Monoclinic, $P 2_{1} / n$ \\
\hline Temperature $(\mathrm{K})$ & 153 \\
\hline$a, b, c(\AA)$ & $7.5555(3), 13.0278(7), 9.7397$ (5) \\
\hline$\beta\left(^{\circ}\right)$ & $91.767(2)$ \\
\hline$V\left(\AA^{3}\right)$ & $958.24(8)$ \\
\hline$Z$ & 4 \\
\hline Radiation type & Мо $K \alpha$ \\
\hline$\mu\left(\mathrm{mm}^{-1}\right)$ & 0.36 \\
\hline Crystal size $(\mathrm{mm})$ & $0.60 \times 0.60 \times 0.20$ \\
\hline \multicolumn{2}{|l|}{ Data collection } \\
\hline Diffractometer & Bruker $\mathrm{X} 8$ APEX2 CCD detector \\
\hline Absorption correction & $\begin{array}{l}\text { Multi-scan (SADABS; Bruker, } \\
\text { 2008) }\end{array}$ \\
\hline$T_{\min }, T_{\max }$ & $0.814,0.932$ \\
\hline $\begin{array}{l}\text { No. of measured, independent and } \\
\text { observed }[I>2 \sigma(I)] \text { reflections }\end{array}$ & $8464,1988,1795$ \\
\hline$R_{\text {int }}$ & 0.021 \\
\hline$(\sin \theta / \lambda)_{\max }\left(\AA^{-1}\right)$ & 0.628 \\
\hline \multicolumn{2}{|l|}{ Refinement } \\
\hline$R\left[F^{2}>2 \sigma\left(F^{2}\right)\right], w R\left(F^{2}\right), S$ & $0.027,0.074,1.07$ \\
\hline No. of reflections & 1988 \\
\hline No. of parameters & 121 \\
\hline $\mathrm{H}$-atom treatment & $\mathrm{H}$-atom parameters constrained \\
\hline$\Delta \rho_{\max }, \Delta \rho_{\min }\left(\mathrm{e} \AA^{-3}\right)$ & $0.22,-0.26$ \\
\hline
\end{tabular}

Computer programs: APEX2 and SAINT (Bruker, 2008), SHELXS97 and SHELXTL (Sheldrick, 2008), SHELXL2015 (Sheldrick, 2015) and ORTEP-3 for Windows (Farrugia, 2012).

\section{Refinement}

Crystal data, data collection and structure refinement details are summarized in Table $2 . \mathrm{H}$ atoms were included in calculated positions $(\mathrm{C}-\mathrm{H}=0.95,0.98 \AA ; \mathrm{O}-\mathrm{H}=0.84 \AA$ ) and allowed to ride on their parent atoms with $U_{\text {iso }}(\mathrm{H})=$ $1.5 U_{\text {eq }}(\mathrm{C}, \mathrm{O})$ for methyl and hydroxy $\mathrm{H}$ atoms and $1.2 U_{\text {eq }}(\mathrm{C})$ for aryl $\mathrm{H}$ atoms.

\section{Funding information}

This work was supported by the Deutsche Forschungsgemeinschaft (DFG) under the program SPP 1362/1 and by the European Union (European Regional Development Fund) including the Ministry of Science and Art of Saxony (Cluster of Excellence "Structure Design of Novel High-Performance Materials via Atomic Design and Defect Engineering, ADDE").

\section{References}

Bourne, S. A., Caira, M. R., Nassimbeni, L. R., Sakamoto, M., Tanaka, K. \& Toda, F. (1994). J. Chem. Soc. Perkin Trans. 2, pp. 1899-1900. Bruker (2008). APEX2, SAINT and SADABS. Bruker AXS Inc., Madison, Wisconsin, USA.

Desiraju, G. R. \& Steiner, T. (1999). The Weak Hydrogen Bond. IUCr Monographs on Crystallography, Vol. 9, ch. 3, p. 164. Oxford University Press.

Desiraju, G. R. , Vittal, G. G. \& Ramonau, A. (2012). In Crystal Engineering. London: Imperial College Press.

Farrugia, L. J. (2012). J. Appl. Cryst. 45, 849-854.

Groom, C. R., Bruno, I. J., Lightfoot, M. P. \& Ward, S. C. (2016). Acta Cryst. B72, 171-179.

Günthel, M., Hübscher, J., Dittrich, R., Weber, E., Joseph, I. \& Mertens, F. (2015). J. Polym. Sci. Part B Polym. Phys. 53, 335-344.

Hübscher, J., Seichter, W., Gruber, T., Kortus, J. \& Weber, E. (2015). J. Heterocycl. Chem. 52, 1062-1074.

Hübscher, J., Seichter, W. \& Weber, E. (2017). CrystEngComm, 19, 3026-3036.

MacGillivray, L. R. (2010). In Metal-Organic Frameworks. Hoboken: Wiley.

Mukherjee, A., Tothadi, S. \& Desiraju, G. R. (2014). Acc. Chem. Res. 47, 2514-2524.

Noro, S.-I. \& Kitagawa, S. (2010). The Supramolecular Chemistry of Organic Hybrid Materials, edited by K. Rurack \& R. MartinezMánez, pp. 235-269. Chichester: Wiley.

Pérez-Balado, C., Ormerod, D., Aelterman, W. \& Mertens, N. (2007). Org. Process Res. Dev. 11, 237-240.

Sheldrick, G. M. (2008). Acta Cryst. A64, 112-122.

Sheldrick, G. M. (2015). Acta Cryst. C71, 3-8.

Sonogashira, K., Tohda, Y. \& Hagihara, N. (1975). Tetrahedron Lett. 16, 4467-4470.

Tiekink, E. R. T. \& Zukerman-Schpector, J. (2012). In The Importance of Pi-Interactions in Crystal Engineering. Frontiers in Crystal Engineering. Chichester: Wiley.

Toda, F., Tanaka, K., Ueda, H. \& Oshima, T. (1983). J. Chem. Soc. Chem. Commun. pp. 743-744.

Toda, F., Tanaka, K., Ueda, H. \& Ōshima, T. (1985). Isr. J. Chem. 25, 338-345.

Wang, L.-C. \& Zheng, Q.-Y. (2015). In Hydrogen Bonded Supramolecular Structures. Lecture Notes in Supramolecular Chemistry, edited by Z.-T. Li \& L. Z. Wu, Vol. 87, pp. 69-113. Berlin, Heidelberg: Springer. 


\section{supporting information}

Acta Cryst. (2017). E73, 1172-1174 [https://doi.org/10.1107/S2056989017010027]

\section{Crystal structure of 2-chloro-5-(3-hydroxy-3-methyllout-1-yn-1-yl)pyrimidine}

\section{Jörg Hübscher, Wilhelm Seichter and Edwin Weber}

\section{Computing details}

Data collection: APEX2 (Bruker, 2008); cell refinement: SAINT (Bruker, 2008); data reduction: SAINT (Bruker, 2008); program(s) used to solve structure: SHELXS97 (Sheldrick, 2008); program(s) used to refine structure: SHELXL2015 (Sheldrick, 2015); molecular graphics: ORTEP-3 for Windows (Farrugia, 2012); software used to prepare material for publication: SHELXTL (Sheldrick, 2008).

2-Chloro-5-(3-hydroxy-3-methylbut-1-yn-1-yl)pyrimidine

Crystal data

$\mathrm{C}_{9} \mathrm{H}_{9} \mathrm{ClN}_{2} \mathrm{O}$

$M_{r}=196.63$

Monoclinic, $P 2_{1} / n$

$a=7.5555(3) \AA$

$b=13.0278$ (7) $\AA$

$c=9.7397(5) \AA$

$\beta=91.767(2)^{\circ}$

$V=958.24(8) \AA^{3}$

$Z=4$

Data collection

Bruker X8 APEX2 CCD detector diffractometer

$\varphi$ and $\omega$ scans

Absorption correction: multi-scan

(SADABS; Bruker, 2008)

$T_{\text {min }}=0.814, T_{\max }=0.932$

8464 measured reflections

\section{Refinement}

Refinement on $F^{2}$

Least-squares matrix: full

$R\left[F^{2}>2 \sigma\left(F^{2}\right)\right]=0.027$

$w R\left(F^{2}\right)=0.074$

$S=1.07$

1988 reflections

121 parameters

0 restraints
$F(000)=408$

$D_{\mathrm{x}}=1.363 \mathrm{Mg} \mathrm{m}^{-3}$

Mo $K \alpha$ radiation, $\lambda=0.71073 \AA$

Cell parameters from 5586 reflections

$\theta=2.6-29.2^{\circ}$

$\mu=0.36 \mathrm{~mm}^{-1}$

$T=153 \mathrm{~K}$

Block, colourless

$0.60 \times 0.60 \times 0.20 \mathrm{~mm}$

1988 independent reflections

1795 reflections with $I>2 \sigma(I)$

$R_{\text {int }}=0.021$

$\theta_{\text {max }}=26.5^{\circ}, \theta_{\min }=2.6^{\circ}$

$h=-9 \rightarrow 9$

$k=-15 \rightarrow 16$

$l=-10 \rightarrow 12$

Hydrogen site location: inferred from neighbouring sites

$\mathrm{H}$-atom parameters constrained

$w=1 /\left[\sigma^{2}\left(F_{\mathrm{o}}^{2}\right)+(0.0363 P)^{2}+0.3033 P\right]$

where $P=\left(F_{\mathrm{o}}{ }^{2}+2 F_{\mathrm{c}}{ }^{2}\right) / 3$

$(\Delta / \sigma)_{\max }<0.001$

$\Delta \rho_{\max }=0.22 \mathrm{e} \AA^{-3}$

$\Delta \rho_{\min }=-0.26$ e $\AA^{-3}$ 


\section{Special details}

Experimental. The melting point was measured using a microscope heating stage (Thermovar, Reichert-Jung). The NMR spectra were obtained on a Bruker Avance $500.1\left({ }^{1} \mathrm{H}\right)$ and $125.8 \mathrm{MHz}\left({ }^{13} \mathrm{C}\right)$ with TMS as internal standard $(\delta$ in $\mathrm{ppm}$ ). The IR spectrum was determined on a Nicolet FT-IR 510 spectrometer as KBr pellet (wavenumber is given in $\mathrm{cm}^{-1}$ ). The mass spectrum was recorded on a Hewlett-Packard 5890 Series II/MS 5989A. Elemental analysis was carried out with a Hanau vario MICRO cube.

Geometry. All esds (except the esd in the dihedral angle between two 1.s. planes) are estimated using the full covariance matrix. The cell esds are taken into account individually in the estimation of esds in distances, angles and torsion angles; correlations between esds in cell parameters are only used when they are defined by crystal symmetry. An approximate (isotropic) treatment of cell esds is used for estimating esds involving 1.s. planes.

Fractional atomic coordinates and isotropic or equivalent isotropic displacement parameters $\left(\AA^{2}\right)$

\begin{tabular}{lllll}
\hline & $x$ & $y$ & $z$ & $U_{\text {iso }} / U_{\text {eq }}$ \\
\hline C11 & $0.23192(4)$ & $0.39748(3)$ & $0.09166(3)$ & $0.03135(12)$ \\
O1 & $0.40922(11)$ & $-0.02601(6)$ & $0.82873(9)$ & $0.0229(2)$ \\
H1 & 0.4836 & 0.0217 & 0.8368 & $0.034^{*}$ \\
N1 & $0.33701(13)$ & $0.23553(8)$ & $0.22655(10)$ & $0.0237(2)$ \\
N2 & $0.14057(13)$ & $0.34952(8)$ & $0.33846(10)$ & $0.0227(2)$ \\
C1 & $0.23671(15)$ & $0.31831(9)$ & $0.23470(12)$ & $0.0205(2)$ \\
C2 & $0.14420(15)$ & $0.28822(9)$ & $0.44881(12)$ & $0.0219(3)$ \\
H2 & 0.0768 & 0.3066 & 0.5258 & $0.026^{*}$ \\
C3 & $0.24367(15)$ & $0.19835(9)$ & $0.45432(12)$ & $0.0196(2)$ \\
C4 & $0.34057(16)$ & $0.17568(9)$ & $0.33843(12)$ & $0.0228(3)$ \\
H4 & 0.4117 & 0.1155 & 0.3389 & $0.027^{*}$ \\
C5 & $0.24486(15)$ & $0.13360(10)$ & $0.57316(12)$ & $0.0222(3)$ \\
C6 & $0.24267(15)$ & $0.08049(9)$ & $0.67312(12)$ & $0.0221(3)$ \\
C7 & $0.23766(15)$ & $0.01625(9)$ & $0.79857(12)$ & $0.0205(2)$ \\
C8 & $0.11447(17)$ & $-0.07507(11)$ & $0.77342(14)$ & $0.0292(3)$ \\
H8A & 0.1610 & -0.1182 & 0.7004 & $0.044^{*}$ \\
H8B & -0.0037 & -0.0503 & 0.7457 & $0.044^{*}$ \\
H8C & 0.1070 & -0.1154 & 0.8580 & $0.044^{*}$ \\
C9 & $0.17864(17)$ & $0.08197(11)$ & $0.91878(13)$ & $0.0281(3)$ \\
H9A & 0.1825 & 0.0408 & 1.0030 & $0.042^{*}$ \\
H9B & 0.0574 & 0.1062 & 0.9003 & $0.042^{*}$ \\
H9C & 0.2581 & 0.1410 & 0.9300 & $0.042^{*}$ \\
& & & &
\end{tabular}

Atomic displacement parameters $\left(\AA^{2}\right)$

\begin{tabular}{lllllll}
\hline & $U^{11}$ & $U^{22}$ & $U^{33}$ & $U^{12}$ & $U^{13}$ & $U^{23}$ \\
\hline C11 & $0.03714(19)$ & $0.0336(2)$ & $0.02359(18)$ & $0.00504(13)$ & $0.00606(13)$ & $0.01072(12)$ \\
O1 & $0.0209(4)$ & $0.0193(4)$ & $0.0288(5)$ & $0.0002(3)$ & $0.0028(3)$ & $0.0023(3)$ \\
N1 & $0.0271(5)$ & $0.0234(5)$ & $0.0209(5)$ & $0.0017(4)$ & $0.0062(4)$ & $-0.0002(4)$ \\
N2 & $0.0255(5)$ & $0.0229(5)$ & $0.0197(5)$ & $0.0039(4)$ & $0.0020(4)$ & $0.0003(4)$ \\
C1 & $0.0221(5)$ & $0.0213(6)$ & $0.0180(6)$ & $-0.0013(4)$ & $0.0010(4)$ & $0.0017(4)$ \\
C2 & $0.0238(6)$ & $0.0246(6)$ & $0.0174(6)$ & $0.0019(5)$ & $0.0031(4)$ & $-0.0024(5)$ \\
C3 & $0.0211(5)$ & $0.0200(6)$ & $0.0176(6)$ & $-0.0021(4)$ & $0.0003(4)$ & $-0.0005(4)$ \\
C4 & $0.0265(6)$ & $0.0194(6)$ & $0.0228(6)$ & $0.0024(5)$ & $0.0043(5)$ & $-0.0004(5)$ \\
C5 & $0.0228(6)$ & $0.0224(6)$ & $0.0215(6)$ & $0.0003(5)$ & $0.0033(5)$ & $-0.0015(5)$
\end{tabular}




\begin{tabular}{lllllll} 
C6 & $0.0231(6)$ & $0.0222(6)$ & $0.0213(6)$ & $0.0007(5)$ & $0.0041(5)$ & $-0.0007(5)$ \\
C7 & $0.0202(5)$ & $0.0224(6)$ & $0.0189(6)$ & $-0.0001(4)$ & $0.0032(4)$ & $0.0023(5)$ \\
C8 & $0.0298(6)$ & $0.0314(7)$ & $0.0264(6)$ & $-0.0090(5)$ & $0.0016(5)$ & $0.0032(5)$ \\
C9 & $0.0287(6)$ & $0.0330(7)$ & $0.0230(6)$ & $0.0040(5)$ & $0.0075(5)$ & $0.0000(5)$ \\
\hline
\end{tabular}

Geometric parameters $\left(\AA,^{\circ}\right)$

\begin{tabular}{|c|c|c|c|}
\hline $\mathrm{C} 11-\mathrm{C} 1$ & $1.7329(12)$ & $\mathrm{C} 4-\mathrm{H} 4$ & 0.9500 \\
\hline $\mathrm{O} 1-\mathrm{C} 7$ & $1.4304(14)$ & $\mathrm{C} 5-\mathrm{C} 6$ & $1.1950(18)$ \\
\hline $\mathrm{O} 1-\mathrm{H} 1$ & 0.8400 & $\mathrm{C} 6-\mathrm{C} 7$ & $1.4824(16)$ \\
\hline $\mathrm{N} 1-\mathrm{C} 1$ & $1.3219(16)$ & $\mathrm{C} 7-\mathrm{C} 8$ & $1.5257(17)$ \\
\hline $\mathrm{N} 1-\mathrm{C} 4$ & $1.3396(16)$ & $\mathrm{C} 7-\mathrm{C} 9$ & $1.5280(17)$ \\
\hline $\mathrm{N} 2-\mathrm{C} 1$ & $1.3265(16)$ & $\mathrm{C} 8-\mathrm{H} 8 \mathrm{~A}$ & 0.9800 \\
\hline $\mathrm{N} 2-\mathrm{C} 2$ & $1.3386(15)$ & $\mathrm{C} 8-\mathrm{H} 8 \mathrm{~B}$ & 0.9800 \\
\hline $\mathrm{C} 2-\mathrm{C} 3$ & $1.3914(17)$ & $\mathrm{C} 8-\mathrm{H} 8 \mathrm{C}$ & 0.9800 \\
\hline $\mathrm{C} 2-\mathrm{H} 2$ & 0.9500 & C9-H9A & 0.9800 \\
\hline $\mathrm{C} 3-\mathrm{C} 4$ & $1.3958(17)$ & $\mathrm{C} 9-\mathrm{H} 9 \mathrm{~B}$ & 0.9800 \\
\hline $\mathrm{C} 3-\mathrm{C} 5$ & $1.4320(16)$ & $\mathrm{C} 9-\mathrm{H} 9 \mathrm{C}$ & 0.9800 \\
\hline $\mathrm{C} 7-\mathrm{O} 1-\mathrm{H} 1$ & 109.5 & $\mathrm{O} 1-\mathrm{C} 7-\mathrm{C} 8$ & $106.10(10)$ \\
\hline $\mathrm{C} 1-\mathrm{N} 1-\mathrm{C} 4$ & $115.04(10)$ & $\mathrm{C} 6-\mathrm{C} 7-\mathrm{C} 8$ & $109.81(10)$ \\
\hline $\mathrm{C} 1-\mathrm{N} 2-\mathrm{C} 2$ & $115.51(10)$ & $\mathrm{O} 1-\mathrm{C} 7-\mathrm{C} 9$ & $110.02(9)$ \\
\hline $\mathrm{N} 1-\mathrm{C} 1-\mathrm{N} 2$ & $128.63(11)$ & $\mathrm{C} 6-\mathrm{C} 7-\mathrm{C} 9$ & $109.31(10)$ \\
\hline $\mathrm{N} 1-\mathrm{C} 1-\mathrm{C} 11$ & $115.79(9)$ & $\mathrm{C} 8-\mathrm{C} 7-\mathrm{C} 9$ & $111.65(10)$ \\
\hline $\mathrm{N} 2-\mathrm{C} 1-\mathrm{C} 11$ & $115.58(9)$ & $\mathrm{C} 7-\mathrm{C} 8-\mathrm{H} 8 \mathrm{~A}$ & 109.5 \\
\hline $\mathrm{N} 2-\mathrm{C} 2-\mathrm{C} 3$ & $122.05(11)$ & $\mathrm{C} 7-\mathrm{C} 8-\mathrm{H} 8 \mathrm{~B}$ & 109.5 \\
\hline $\mathrm{N} 2-\mathrm{C} 2-\mathrm{H} 2$ & 119.0 & $\mathrm{H} 8 \mathrm{~A}-\mathrm{C} 8-\mathrm{H} 8 \mathrm{~B}$ & 109.5 \\
\hline $\mathrm{C} 3-\mathrm{C} 2-\mathrm{H} 2$ & 119.0 & $\mathrm{C} 7-\mathrm{C} 8-\mathrm{H} 8 \mathrm{C}$ & 109.5 \\
\hline $\mathrm{C} 2-\mathrm{C} 3-\mathrm{C} 4$ & $116.30(11)$ & $\mathrm{H} 8 \mathrm{~A}-\mathrm{C} 8-\mathrm{H} 8 \mathrm{C}$ & 109.5 \\
\hline $\mathrm{C} 2-\mathrm{C} 3-\mathrm{C} 5$ & $121.11(11)$ & $\mathrm{H} 8 \mathrm{~B}-\mathrm{C} 8-\mathrm{H} 8 \mathrm{C}$ & 109.5 \\
\hline $\mathrm{C} 4-\mathrm{C} 3-\mathrm{C} 5$ & $122.59(11)$ & $\mathrm{C} 7-\mathrm{C} 9-\mathrm{H} 9 \mathrm{~A}$ & 109.5 \\
\hline $\mathrm{N} 1-\mathrm{C} 4-\mathrm{C} 3$ & $122.47(11)$ & $\mathrm{C} 7-\mathrm{C} 9-\mathrm{H} 9 \mathrm{~B}$ & 109.5 \\
\hline $\mathrm{N} 1-\mathrm{C} 4-\mathrm{H} 4$ & 118.8 & $\mathrm{H} 9 \mathrm{~A}-\mathrm{C} 9-\mathrm{H} 9 \mathrm{~B}$ & 109.5 \\
\hline $\mathrm{C} 3-\mathrm{C} 4-\mathrm{H} 4$ & 118.8 & $\mathrm{C} 7-\mathrm{C} 9-\mathrm{H} 9 \mathrm{C}$ & 109.5 \\
\hline $\mathrm{C} 6-\mathrm{C} 5-\mathrm{C} 3$ & $178.65(13)$ & $\mathrm{H} 9 \mathrm{~A}-\mathrm{C} 9-\mathrm{H} 9 \mathrm{C}$ & 109.5 \\
\hline $\mathrm{C} 5-\mathrm{C} 6-\mathrm{C} 7$ & $178.79(13)$ & $\mathrm{H} 9 \mathrm{~B}-\mathrm{C} 9-\mathrm{H} 9 \mathrm{C}$ & 109.5 \\
\hline $\mathrm{O} 1-\mathrm{C} 7-\mathrm{C} 6$ & $109.91(9)$ & & \\
\hline $\mathrm{C} 4-\mathrm{N} 1-\mathrm{C} 1-\mathrm{N} 2$ & $0.20(19)$ & $\mathrm{N} 2-\mathrm{C} 2-\mathrm{C} 3-\mathrm{C} 4$ & $0.30(17)$ \\
\hline $\mathrm{C} 4-\mathrm{N} 1-\mathrm{C} 1-\mathrm{C} 11$ & $179.40(9)$ & $\mathrm{N} 2-\mathrm{C} 2-\mathrm{C} 3-\mathrm{C} 5$ & $-179.66(11)$ \\
\hline $\mathrm{C} 2-\mathrm{N} 2-\mathrm{C} 1-\mathrm{N} 1$ & $-0.89(19)$ & $\mathrm{C} 1-\mathrm{N} 1-\mathrm{C} 4-\mathrm{C} 3$ & $0.82(17)$ \\
\hline $\mathrm{C} 2-\mathrm{N} 2-\mathrm{C} 1-\mathrm{Cl} 1$ & $179.91(8)$ & $\mathrm{C} 2-\mathrm{C} 3-\mathrm{C} 4-\mathrm{N} 1$ & $-1.05(18)$ \\
\hline $\mathrm{C} 1-\mathrm{N} 2-\mathrm{C} 2-\mathrm{C} 3$ & $0.58(17)$ & $\mathrm{C} 5-\mathrm{C} 3-\mathrm{C} 4-\mathrm{N} 1$ & $178.91(11)$ \\
\hline
\end{tabular}

Hydrogen-bond geometry $\left(\AA,{ }^{\circ}\right)$

\begin{tabular}{lllll}
\hline$D-\mathrm{H} \cdots A$ & $D-\mathrm{H}$ & $\mathrm{H} \cdots A$ & $D \cdots A$ & $D-\mathrm{H} \cdots A$ \\
\hline $\mathrm{C} 8-\mathrm{H} 8 A \cdots \mathrm{C} 11^{\mathrm{i}}$ & 0.98 & 2.99 & $3.7911(14)$ & 140
\end{tabular}


supporting information

$\begin{array}{lllll}\mathrm{C} 4-\mathrm{H} 4 \cdots \mathrm{O} 1^{\mathrm{ii}} & 0.95 & 2.45 & 3.1963(15) & 136 \\ \mathrm{C} 2-\mathrm{H} 2 \cdots \mathrm{O} 1^{\mathrm{iii}} & 0.95 & 2.60 & 3.2816(14) & 129 \\ \mathrm{O} 1-\mathrm{H} 1 \cdots \mathrm{N} 2^{\mathrm{iv}} & 0.84 & 2.05 & 2.8881(13) & 172\end{array}$

Symmetry codes: (i) $-x+1 / 2, y-1 / 2,-z+1 / 2$; (ii) $-x+1,-y,-z+1$; (iii) $-x+1 / 2, y+1 / 2,-z+3 / 2$; (iv) $x+1 / 2,-y+1 / 2, z+1 / 2$. 